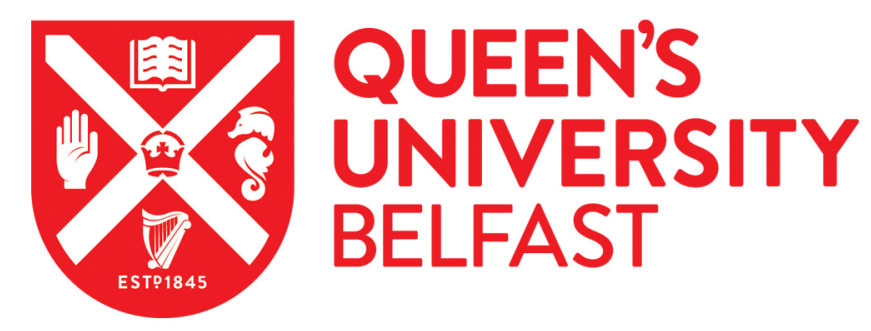

\title{
Reduced synthesis in harmonic analysis and compact synthesis in operator theory
}

Shulman, V. S., Todorov, I. G., \& Turowska, L. (2017). Reduced synthesis in harmonic analysis and compact synthesis in operator theory. Functional Analysis and Its Applications, 51(3), 240-243.

https://doi.org/10.1007/s10688-017-0189-9

Published in:

Functional Analysis and Its Applications

Document Version:

Peer reviewed version

Queen's University Belfast - Research Portal:

Link to publication record in Queen's University Belfast Research Portal

Publisher rights

(c) 2017 Springer Science+Business Media, LLC.

This work is made available online in accordance with the publisher's policies. Please refer to any applicable terms of use of the publisher.

\section{General rights}

Copyright for the publications made accessible via the Queen's University Belfast Research Portal is retained by the author(s) and / or other copyright owners and it is a condition of accessing these publications that users recognise and abide by the legal requirements associated with these rights.

Take down policy

The Research Portal is Queen's institutional repository that provides access to Queen's research output. Every effort has been made to ensure that content in the Research Portal does not infringe any person's rights, or applicable UK laws. If you discover content in the Research Portal that you believe breaches copyright or violates any law, please contact openaccess@qub.ac.uk. 


\title{
Компактный синтез
}

\author{
И.Г.Тодоров, Л.Туровская и В.С.Шульман
}

\section{1 Ослабленный синтез в гармоническом анализе}

Напомним, вначале, как определяются (см. [3]) некоторые понятия гармонического анализа в случае некоммутативных групп (то есть, в отсутствие дуальной группы).

Пусть $G$ - локально компактная группа. Обозначим через $\lambda: G \rightarrow \mathcal{B}\left(L^{2}(G)\right)$, $s \mapsto \lambda_{s}$, ее левое регулярное представление: $\left(\lambda_{s} \xi\right)(x)=\xi\left(s^{-1} x\right)$. Тем же символом мы обозначаем и регулярное представление алгебры $L^{1}(G)$ на $L^{2}(G)$.

Приведенная групповая $\mathrm{C}^{*}$-алгебра $C_{r}^{*}(G)$ группы $G$ - это замыкание по операторной норме алгебры $\lambda\left(L^{1}(G)\right)=\left\{\lambda(f): f \in L^{1}(G)\right\}$; ее *-слабое замыкание $\operatorname{VN}(G)$ называется алгеброй фон Неймана группы $G$.

Алгеброй Фуръе группы $G$ называется алгебра $A(G)$ всех функций $и: G \rightarrow \mathbb{C}$ допускающих представление вида $u(x)=\left(\lambda_{x} \xi, \eta\right)$, где $\xi, \eta \in L^{2}(G)$. Это коммутативная регулярная банахова алгебра относительно нормы $\|u\|=\inf \{\|\xi\|\|\eta\|\}$. Ее идеалам сопоставляются множества нулей: null $J=\{s \in G: u(s)=0$ для всех $u \in J\}$, и, напротив, замкнутым подмножествам сопоставляются идеалы $I(E)=\{f \in A(G): f(s)=$ $0, s \in E\}, J_{0}(E)=\{f \in A(G): f(s)=0$ в окрестности $E$, носитель $f$ компактен $\}-$ наибольший и наименьший идеалы с множеством нулей $E$. Полагая $J(E)=\overline{J_{0}(E)}$, мы говорим, что $E$ является множеством синтеза, если $I(E)=J(E)$.

Чтобы определить понятие ослабленного синтеза, запишем это равенство, перейдя к аннуляторам в сопряженном пространстве: $I(E)^{\perp}=J(E)^{\perp}$. Пространство, сопряженное к $A(G)$, может быть отождествлено с $\mathrm{VN}(G)$ путем сопоставления каждому оператору $T \in \operatorname{VN}(G)$ функционала $u \mapsto(T \xi, \eta)$, где $u(s)=\left(\lambda_{s} \xi, \eta\right)$. Будем говорить, что замкнутое множество $E \subset G$ допускает ослабленный синтез, если

$$
C_{r}^{*}(G) \cap I(E)^{\perp}=C_{r}^{*}(G) \cap J(E)^{\perp} .
$$

В классическом случае $G=\mathbb{T}$ это означает, что все сосредоточенные на $E$ псевдофункции (распределения, коэффициенты Фурье которых стремятся к нулю), аппроксимируются мерами, сосредоточенными на $E$. В частности, все множества единственности (т.е. не несущие ненулевых псевдофункций) допускают ослабленный синтез. Класс множеств единственности интенсивно изучался, поскольку он играет важную роль в теории тригонометрических рядов. В общем случае, говорят, что $E$ является множеством единственности (или $U$-множеством), если $C_{r}^{*}(G) \cap J(E)^{\perp}=\{0\}$.

Л.Шварц в знаменитой работе [6] показал, что сфера $S^{n-1}$ не является множеством синтеза в группе $\mathbb{R}^{n}$ при $n \geq 3$. С другой стороны, Варопулос [10] доказал, что $S^{2}$ 
допускает ослабленный синтез в $\mathbb{R}^{3}$. Оказывается, что на большие размерности этот результат не переносится:

noness Tеорема 1.1 (i) При $n \geq 4$ сфера $S^{n-1}$ не является множеством ослабленного синтеза в $\mathbb{R}^{n}$.

(ii) При $n \geq 6$ световой конус $\left\{x \in \mathbb{R}^{n}: x_{1}^{2}+x_{2}^{2}+\ldots+x_{n-1}^{2}-x_{n}^{2}=0\right\}$ не является множеством ослабленного синтеза в $\mathbb{R}^{n}$.

Следующий результат усиливает известную теорему Маявэна [4]. Для коммутативных метризуемых групп он был получен Саеки [5].

Ма Теорема 1.2 Всякая недискретная локально компактная группа, имеющая открытую коммутативную подгруппу, содержит замкнутое множество, не являющееся множеством ослабленного синтеза.

\section{2 Компактный синтез}

Пусть $(X, \mu)$ и $(Y, \nu)$ - стандартные пространства с мерами. Прямоугольниками в $X \times$ $Y$ мы будем называть множества вида $\alpha \times \beta$, где $\alpha \subseteq X$ и $\beta \subseteq Y$ измеримы. Множество называется маргинально нулевым, если оно содержится в $(\alpha \times Y) \cup(X \times \beta)$, где $\alpha, \beta$ имеют нулевую меру. Подмножества $E, F \subseteq X \times Y$ маргинально эквивалентны $(E \simeq$ $F)$, если их симметрическая разность маргинально нулевая. Подмножество $E \subset X \times Y$ называется $\omega$-открытым, если оно маргинально эквивалентно счетному объединению прямоугольников. Дополнения к $\omega$-открытым множествам называются $\omega$-замкнутыми $[2]$.

Пусть $H_{1}=L_{2}(X, \mu)$ и $H_{2}=L_{2}(Y, \nu)$. Через $\mathcal{B}\left(H_{1}, H_{2}\right)$ обозначается пространство ограниченных линейных операторов из $H_{1}$ в $H_{2}$, а через $\mathcal{K}\left(H_{1}, H_{2}\right)\left(\mathcal{C}_{p}\left(H_{1}, H_{2}\right)\right)-$ пространство компактных операторов (соответственно, идеалы Шэттена).

Оператор умножения на $f \in L^{\infty}(X, \mu)$ в $L^{2}(X, \mu)$ обозначается $M_{f}$. Множество всех таких операторов образует максимальную абелеву самосопряженную алгебру операторов (masa) на $L^{2}(X, \mu)$. Для измеримого множества $\alpha \subseteq X$ мы обозначаем через $\chi_{\alpha}$ его характеристическую функцию и полагаем $P(\alpha)=M_{\chi_{\alpha}}$. Подпространство $\mathcal{W} \subseteq \mathcal{B}\left(H_{1}, H_{2}\right)$ называется таsa-бимодулем, если $M_{\psi} T M_{\varphi} \in \mathcal{W}$ для всех $T \in \mathcal{W}$, $\varphi \in L^{\infty}(X, \mu)$ и $\psi \in L^{\infty}(Y, \nu)$.

Скажем, что $\omega$-замкнутое подмножество $E \subseteq X \times Y$ несет оператор $T \in \mathcal{B}\left(H_{1}, H_{2}\right)$ (или $T$ сосредоточен на $E$ ), если $P(\beta) T P(\alpha)=0$, когда $\alpha \times \beta \cap E=\emptyset$. У любого $\mathcal{M} \subseteq$ $\mathcal{B}\left(H_{1}, H_{2}\right)$, существует носитель, то есть наименьшее (с точностью до маргинальной эквивалентности) $\omega$-замкнутое множество $\operatorname{supp} \mathcal{M}$, несущее все операторы из $\mathcal{M}[2]$.

Для фиксированного $E$ множество $\mathfrak{M}_{\max }(E)$ всех операторов, сосредоточенных на $E$, является $w^{*}$-замкнутым masa-бимодулем с носителем $E$. Оно является наибольшим из бимодулей с этими свойствами (откуда и обозначение). Согласно [8], 
существует и наименьший $w^{*}$-замкнутый masa-бимодуль с носителем $E$, он обозначается $\mathfrak{M}_{\min }(E)$. Если $\mathfrak{M}_{\min }(E)=\mathfrak{M}_{\max }(E)$, то, по определению, E допускает операторный синтез. Если же выполнено более слабое условие $\mathfrak{M}_{\text {min }}(E) \cap \mathcal{K}\left(H_{1}, H_{2}\right)=$ $\mathfrak{M}_{\text {max }}(E) \cap \mathcal{K}\left(H_{1}, H_{2}\right)$, то E допускает компактный синтез. Аналогично определяются $\mathcal{C}_{p}\left(H_{1}, H_{2}\right)$-синтезируемые множества.

Примерами компактно-синтезируемых множеств являются операторные $U$-множества, изучению которых посвящена работа [7].

Теорема 2.1 Пусть $G$ - локально компактная группа со второй аксиомой счетности, такая что алгебра $A(G)$ имеет (не обязательно ограниченную) аппроксимативную единицу. Замкнутое подмножество $E \subset G$ допускает ослабленный синтез тогда и только тогда, когда множество $E^{*}:=\left\{(x, y):\right.$ y $\left.x^{-1} \in E\right\} \subset G \times G$ допускает компактный синтез (в качестве меры в $G$ рассматривается левая мера Хаара).

Этот результат работает "в обе стороны" - операторный подход часто удобнее спектрального, а результаты и конструкции гармонического анализа получают полезные операторные приложения. В частности, используя теорему 2.1 и теорему 1.1, легко показать, что пересечение двух множеств компактного синтеза может не допускать компактный синтез. Вопрос о компактной синтезируемости объединения двух множеств компактного синтеза открыт, так же как аналогичный вопрос для операторного и для спектрального синтеза (последний - это одна из известнейших проблем гармонического анализа).

Нашей следующей задачей является нахождение удобных критериев компактной синтезируемости.

Предложение 2.2 Пусть $E-\omega$-замкнутое подмножество в $X \times Y$. (i) Eсли любой компактный оператор, сосредоточенный на $E$, является *-слабым пределом операторов Гильберта-Шмидта, сосредоточенных на $E$, то Е является множеством компактного синтеза.

(ii) При условии, что Е является ш-замыканием своей обратное; более того, из компактной синтезируемости следует в этом случае, что $\mathfrak{M}_{\text {mах }}(E) \cap \mathcal{K}\left(H_{1}, H_{2}\right)$ является замкнутой по операторной норме линейной оболочкой операторов ранга 1, сосредоточенных на $E$.

Чтобы получить "структурные" критерии компактной синтезируемости, введем некоторые определения.

Подмножество $Q \subset X \times Y$ называется элементарным, если оно является объединением конечного числа прямоугольников (их можно считать непересекающимися): $Q=\sum_{i=1}^{n} \Pi_{i}, \quad \Pi_{i}=A_{i} \times B_{i}$. Такое множество определяет проектор $T \mapsto \sum_{i=1}^{n} P_{A_{i}} T P_{B_{i}}$ в $\mathcal{B}\left(H_{1}, H_{2}\right)$, который мы обозначим $\pi_{Q}$. Его образом является $\mathfrak{M}_{\text {max }}(Q)$.

Будем называть множество $E \subset X \times Y$ тонким, если оно является пересечением убывающей последовательности элементарных множеств $\left\{Q_{n}\right\}_{n=1}^{\infty}$, такой что 
$\left\|\pi_{Q_{n}}(T)\right\| \rightarrow 0$ для любого компактного оператора $T$. Множество, представимое в виде объединения тонкого и $\omega$-открытого множества, называется множеством с тонкой границей.

Следующая теорема устанавливает, что множества с тонкой границей образуют "стабилизирующий" подкласс в классе множеств компактного синтеза.

Теорема 2.3 Если $\tau \subset X \times Y-$ множество компактного синтеза, а $E-$ множество с тонкой границей, то их обгединение и пересечение - множества компактного синтеза.

Множества вида $E=\left\{(x, y) \in X \times Y: f_{j}(x) \leq g_{j}(y), j=1, \ldots, n\right\}$, где $f_{j}: X \rightarrow$ $\mathbb{R}, g_{j}: Y \rightarrow \mathbb{R}$ - измеримые функции, назваются множествами конечной ширины. Известно [8], что множества конечной ширины допускают операторный синтез; этот результат обобщает теорему Арвесона [1] о рефлексивности коммутативных решеток, порожденных конечным числом цепочек.

thinwidth Предложение 2.4 Всякое множество конечной ширины имеет тонкую границу.

Применяя теорему 2.3, мы заключаем, что пересечение (и объединение) множества конечной ширины с множеством компактного синтеза являются множествами компактного синтеза. Прямой аналог этого результата для операторного синтеза (в части пересечений) неверен, как показывает [8, теорема 4.9].

Примеры множеств компактного синтеза, имеющие более сложную структуру, можно получить, используя теорему 2.1 и известные конструкции гармонического анализа.

В заключение укажем одно применение полученных результатов к теории линейных операторных уравнений "Фуглидова типа".

Теорема 2.5 Пусть $p>2$. Для любого натурального числа $m>(24 p-12) /(p-2)$ существуют коммутативные семейства нормальных операторов $\left\{A_{i}\right\}_{i=1}^{m},\left\{B_{i}\right\}_{i=1}^{m} u$ оператор $X \in \mathcal{C}_{p}$, такие что $\sum_{i=1}^{m} A_{i} X B_{i}=0$, но $\sum_{i=1}^{m} A_{i}^{*} X B_{i}^{*} \neq 0$.

Компактный оператор, различающий данные уравнения можно найти, когда $m \geq$ 25.

Заметим, что в классе $\mathcal{C}_{2}$ данные линейные операторные уравнения эквивалентны. Другие достаточные условия эквивалентности этих (и близких к ним) уравнений можно найти в [9].

\section{Список литературы}

arv [1] W.B. ARveson, Operator algebras and invariant subspaces, Ann. Math. (2) 100(1974), 433-532

eks [2] J.A. Erdos, A. Katavolos And V.S. Shulman, Rank one subspaces of Bimodules over Maximal Abelian Selfadjoint Algebras, J. Funct. Anal. 157 No.2 (1998), 554-587 
eym [3] P. Eymard, L'algèbre de Fourier d'un groupe localement compact, Bull. Soc. Math. France 92 (1964), 181-236

Mal [4] P.MAllyavin, Impossibilite de la synthese spectrale sur les groupes abeliens non compacts, Publ. Math. Inst. Hautes Etudes Sci., 1959, p. 85-92

saeki [5] S.SAEKI, Helson sets which disobey spectral synthesis, Proc. Amer. Math. Soc. 47 (1975), 371-377

Schwartz [6] L.SchWARTz, Sur le propriete des synthese spectrale dans les groupes non-compacts, C. R. Acad. Sci. Paris 227(1948), 424-426

gralmul [7] V.S. Shulman, I.G. TOdorov And L. TurowskA, Sets of multiplicity and closable multipliers on group algebras, J.Funct.Anal. 268 (2015), no.6, 1454-1508

st1 [8] V.S. Shulman And L. Turowska, Operator synthesis I. Synthetic sets, bilattices and tensor algebras, J. Funct. Anal. 209 (2004), 293-331

st2 [9] V.S. Shulman And L. Turowska, Operator synthesis II. Individual synthesis and linear operator equations, J. Reine Angew. Math. 590 (2006), 143-187

[10] N.Th. Varopoulos, Spectral synthesis on spheres, Proc. Cambridge Philos. Soc. 62(1966), 379-387.

Pure Mathematics Research Centre, Queen's University Belfast, Belfast BT7 1NN, United Kingdom, i.todorov@qub.ac.uk

Department of Mathematical Sciences, Chalmers University of Technology and the University of Gothenburg, Gothenburg SE-412 96, Sweden, turowska@chalmers.se

кафедра высшей математики, Вологодский Государственный университет, Вологда, Россия, shulman.victor80@gmail.com 